\section{Numerical Study of Characteristics Root Canal Irrigation using Open-Ended Needle Positive Pressure and Negative Pressure Method}

\author{
Fajar Dwi Yudantơ Wawan Aries Widodo, Suwarno Suwarno \\ Department of Mechanical Engineering, Insitut Teknologi Sepuluh Nopember, Surabaya 60111, Indonesia \\ Received: 21 January 2020, Revised: 10 March 2020, Accepted: 19 March 2020
}

\begin{abstract}
This study aims to determine the characteristics of fluid flow through root canal irrigation using positive and negative pressure methods used CFD (Computational Fluid Dynamic) software by modeling root canals and needle insertion in the root canals. This modeling used a steady flow, an incompressible flow, and uniform flow at the inlet side, and the roughness of the root canal wall surface is neglected (smooth wall). The open-ended needle with different insertion lengths applied for each method with a flow rate of $0.2 \mathrm{~mL} / \mathrm{s}$ or about $6.63 \mathrm{~m} / \mathrm{s}$ and the Reynolds number is 1376 (Laminar). The fluid has $2.5 \%$ of $\mathrm{NaOCl}$ with the density of $1060 \mathrm{~kg} / \mathrm{m}^{3}$ and 0.001 Pa.s of fluid viscosity. After considering factors such as velocity, dynamic pressure, and wall shear stress in both methods, a greater velocity value in the apical region is determined by using a positive pressure method. Meanwhile, the negative pressure method shows the ability to lift higher due to shear stress, which is more evenly distributed upwards. However, both methods are qualified for irrigation replacement in the apical region.
\end{abstract}

Keywords: CFD, positive pressure method, negative pressure method, open-ended, laminar

\section{Introduction}

Cleaning teeth crust is very important in the medical world as a facility to clean bacteria, crust, etc [1]. Teeth crust cleaning can be done in the root canal or outside. In the root canal, cleaning is done by spraying with $2.5 \% \mathrm{NaOCl}$ for five times then rinsing with distilled water $\left(\mathrm{H}_{2} \mathrm{O}\right)$. Spraying with these chemicals aims to kill bacteria and dissolve organic and inorganic tissue in the direct contact area, while the mechanical action of the liquid is more effective in irrigation mixtures in the canal's lumen and removal of adhesive biofilm and smear layers in the canal surface.

The positive pressure method with open-ended needles cleaning is only done by spraying it on the root canal and allowed to overflow, whereas, in the negative pressure method, there are several steps, namely spraying and suctioning. In addition, the negative pressure method is more effective at cleaning the root canals than the positive pressure method. In this case, it can be explained by fluid computation methods or by CFD (Computational Fluid Dynamic). Computational fluid dynamic (CFD) is a very useful tool to find out what is happening in the root canal by using mathematical models and computer simulations. CFD studies have been widely applied to study irrigant flow patterns in the root canal system. The reliability of CFD analysis in endodontics has been validated with a root canal model so that the experiments can be combined with high speed and velocimetry analysis. An independent CFD analysis has been applied to test the influence of needle position and needle design for root canal cleaning. Most research on dynamics irrigation is related to syringe-based irrigation.

In general, this research is to prove that the negative pressure method is better than the positive pressure [2]. In the negative pressure method by placing the needle farther away from the apical, the results obtained are cleaner than the positive pressure method where the needle placement is closer than the apical. In addition, this study is also to determine the velocity profile of the root canal, wall shear stress in the canal wall, find out the pressure in the root canal, and optimality each method so that it reaches the apical end by variation the depth of the needle inserted in the root canal.

\section{Method}

\subsection{Root Canal Irrigation}

The root canal is part of the tooth under the enamel contained soft tissue called pulp tissue. The pulp tissue contains many nerve fibers, including arteries, blood vessels, lymph vessels, and connective tissue [3]. Each of these nerves enters the tooth through the tip of the root canal or apical. Teeth have at least 1 root canal and a maximum of 4 . Root canal treatment is a non-surgical approach that is used to treat two different things, namely maintaining health around the apical root canal to prevent disease or treat pulp tissue that is dead or infected. Root canal therapy is very necessary because the teeth cannot heal by themselves without treatment; the infection will

"Corresponding author. Email: fajar.yudanto15@mhs.me.its.ac.id 
spread, the bones around the teeth deteriorate until the teeth are separated.

Root canal irrigation is a procedure used to remove the damaged or dead pulp by flushing mechanisms using irrigant fluids such as sodium hypochlorite [4]. Irrigant removes microorganisms, residual tissue, dentinal debris out of the root canal. Cleanup with a dry method that is with no irritation in the root canal before the fluid is injected from the needle or dry conditions. Irrigant must direct contact with the entire canal area to the apical section for optimal effectiveness. After that, the root canal is rinsed again with distilled water $\left(\mathrm{H}_{2} \mathrm{O}\right)$ so that the nature of the acidic fluid is clean from the root canal. Then the root canal is filled with a substance such as rubber called gutta-percha to prevent recontamination and covered with metal alloys [5].

The existing root canal irrigation is still using a needle as a conduit. Generally, root canal irrigation is by the positive pressure method and the negative pressure method. In the positive pressure method, irrigant fluid is injected through the needle and fills the root canal until it moves out. The negative pressure method has suction pressure when cleaning the cone orifice. This suction pressure helps accelerate the movement of irrigation out of the root canal when injected from the needle [6]. The better the irrigant movement, the more effective it is in cleansing because the dead tissue, microorganisms, and dentin can get out of the root canal.

\subsection{Object Geometry}

This numerical study was carried out by simulating a needle inserted into the tooth cone or root canal. The shape of the root canal is frustum and the shape of the needle without changes in diameter [4]. The needles and cones are combined in a way to form a separate geometry between the cone and needle. Needles or syringes inserted into the root canal are called the working length or WL. In addition, the $0 \mathrm{~mm}$ reference is at the base of the root canal or apical. The illustration of the picture can be seen in Figure 1 .

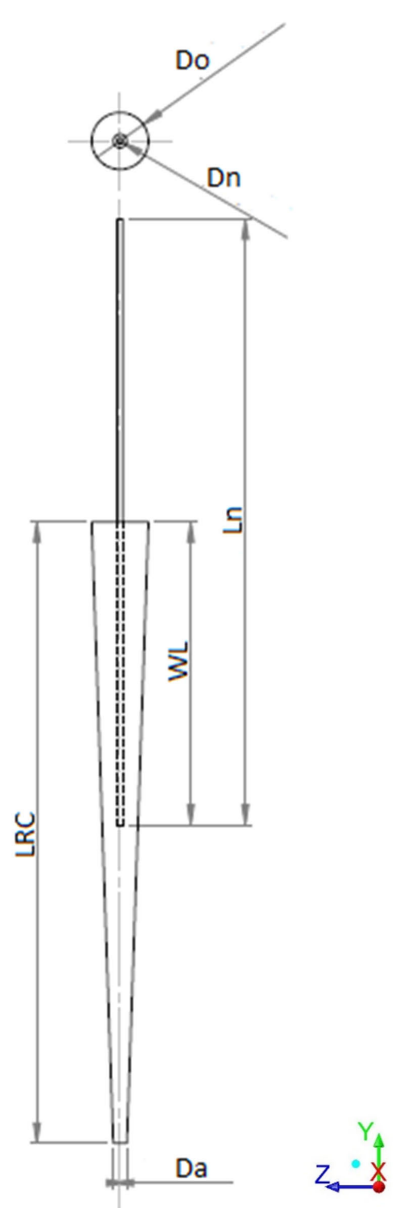

Figure 1. Root canal modeling with open-ended needle positive pressure method (left) and negative pressure method (right). 
The specifications of the geometry of the positive pressure method are as follows:

- Orifice Diameter (Do) : $1.68 \mathrm{~mm}, 1.88 \mathrm{~mm}$, and $1.92 \mathrm{~mm}$

- Apical diameter (Da) : $0.45 \mathrm{~mm}$ (tapper 7\%) [2]

- Syringe diameter(Dn) : $0.196 \mathrm{~mm}$

- Syringe length (Ln) : $25 \mathrm{~mm}$

- Insertion depth (WL) : 2 mm from apical

- Root canal length $\left(\mathrm{L}_{R C}\right)$ : 17.5, 20.5 and $21 \mathrm{~mm}$ (from apical)

The specifications of the geometry of the negative pressure method are as follows:

- Orifice Diameter (Do) : $1.88 \mathrm{~mm}$ and $2.02 \mathrm{~mm}$

- Apical diameter (Da) : 0.45 mm (tapper 7\%) [2]

- Syringe diameter (Dn) : $0.196 \mathrm{~mm}$

- Syringe length (Ln) : $20 \mathrm{~mm}$

- Insertion depth (WL) : $10 \mathrm{~mm}$ from cone orifice

- Root canal length $\left(\mathrm{L}_{R C}\right): 20.5 \mathrm{~mm}$ and $22.5 \mathrm{~mm}$ (from apical)

- Suction pressure : $-20 \mathrm{kPa}$
In the positive pressure method, the variations that will be made including the depth of needle insertion in the root canal and variation of root canal lengths with Reynolds number $\left(\operatorname{Re}_{n}\right)$ value is 1376 . The Reynolds number $\left(\operatorname{Re}_{n}\right)$ value used the same for each variation. Meanwhile, in the negative pressure method, the variation used is the needle distance from the apical, which aims to determine the farthest distance that can be reached by the flow of $0.2 \mathrm{~mL} / \mathrm{s}$ and uses the constant value $\mathrm{Re}_{n}$ of 1376 (Laminar).

\subsection{Numerical Method}

This study simulates the process of root canal irrigation to determine fluid velocity. The simulation in this study utilizes computational fluid dynamic (CFD) to approach numerically and make model geometries. In the modeling, the model is drawn 3D to be like the geometry in Figure 2 and discusses the model (meshing) so the model can be processed with CFD. In general, there will be two stages in numerical research, namely the preprocessing stage and continued with the post-processing stage. In addition, boundary conditions can be seen in Figure 3

\subsection{Data Collection}

The appearance of results after iteration; these results are quantitative and qualitative data. Quantitative data are in the form of distributions of velocity values, shear stresses, and so on. Qualitative data are usually in the form of velocity profile appearance, wall shear stress, and pressure in each cross-section and also the appearance of velocity contours and variations used in this study [6].

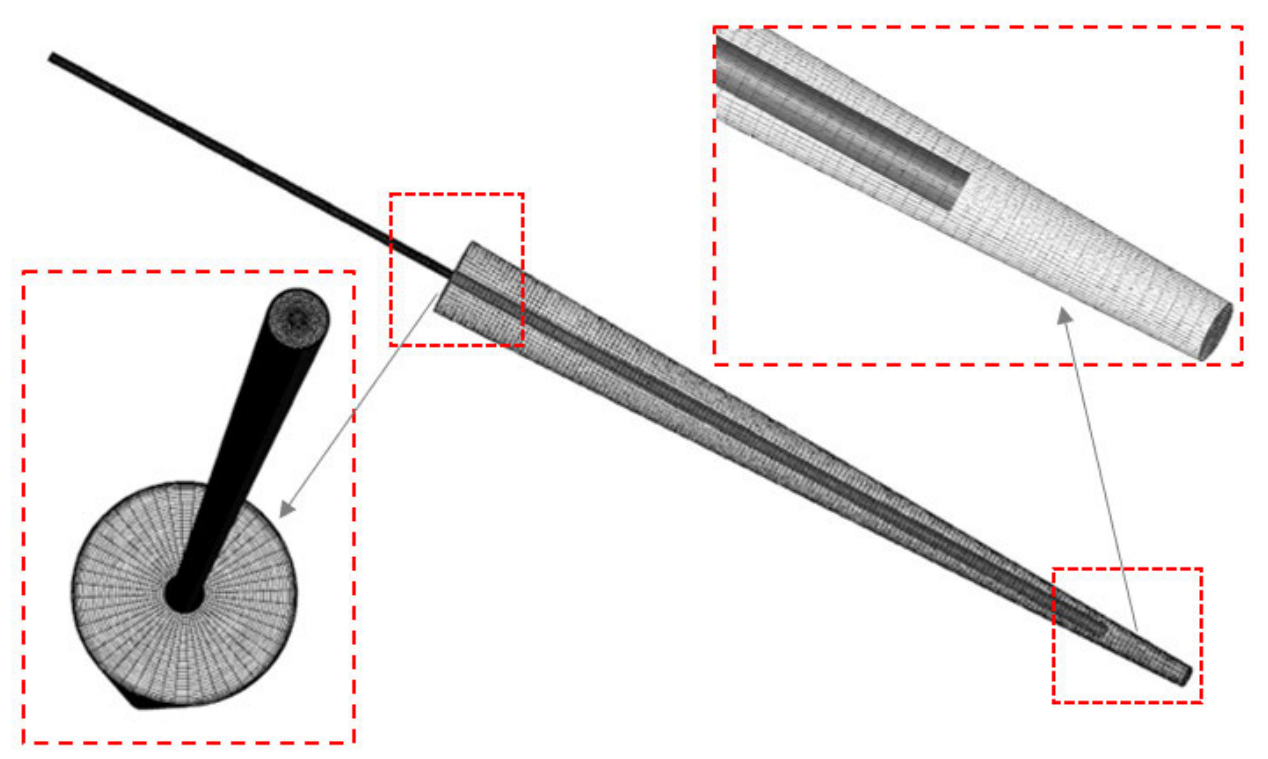

Figure 2. Pre-processing Stage 
In addition to supporting qualitative data (the contours of velocity, shear stress, and dynamic pressure), quantitative data is required. Quantitative data are presented in graphical form. The graph capture position is shown in Figure 4. Retrieval of data is using the line/rake feature. The shear stress is taken in the wall along the root canal, as shown in Figure 4, (Line 1), while for velocity and dynamic pressure, it is taken after the needle exits to the root canal base and from the root canal to the root canal outlet as shown (Line 2). Data collection is done in both methods. Besides, this data collection for the comparison between these two methods is done by drawing a line/rake at the outlet, which then is being used to determine the maximum velocity at Line 3 .

\subsection{Grid Independency}

For the purpose of increasing the accuracy and validity of the model, an all-hexahedral mesh was generated,

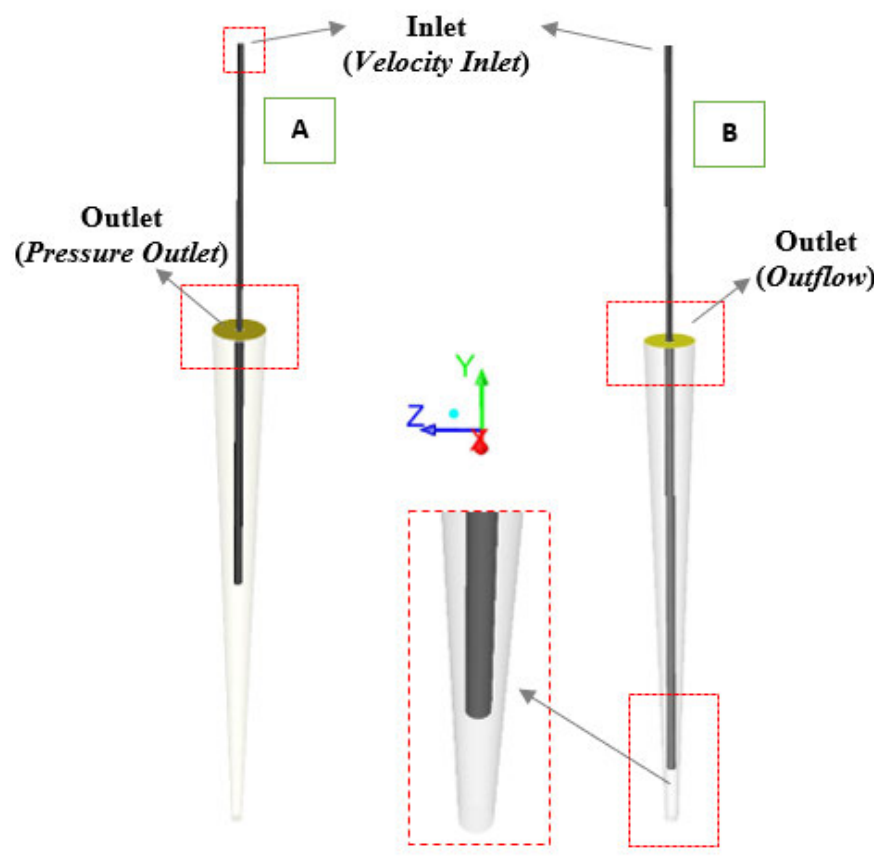

Figure 3. Boundary Condition, (a) Negative Pressure Method and (b) Positive Pressure Method

Table 1. The grid independence is evaluated from $\mathrm{V}_{\text {avg }}$ at the cross-section $\mathrm{y}=1 \mathrm{~mm}$

\begin{tabular}{|c|c|c|c|}
\hline No & Node Amount & Mean Velocity (m/s) & Error (\%) \\
\hline 1 & 488598 & 4.420 & - \\
\hline 2 & 538459 & 4.435 & 0.339 \\
\hline 3 & 580598 & 4.445 & 0.225 \\
\hline 4 & 605918 & 4.447 & 0.044 \\
\hline
\end{tabular}

refined near the walls and in the areas where high gradients of variables were anticipated. To enhance the quality of meshes in the flow channel, the region near curvature surfaces, such as the near-wall regions and flow control devices, were O-type meshed; meanwhile, the far-wall part area was H-type meshed [4]. The grid independency analysis uses the laminar model by analyzing the average velocity $\left(\mathrm{V}_{\text {avg }}\right)$ for each mesh variation at position $\mathrm{y}=$ $1 \mathrm{~mm}$ as reference data, so it can be seen in Table 1 .

From the results of the grid independency, variations in the mesh density were found that there was no significant change in the variation of mesh three with the number of mesh nodes of 580598 nodes. In addition, to clarify the results of the grid independency, a velocity profile at $\mathrm{y}=1 \mathrm{~mm}$ is created for the number of meshes (Figure 5).

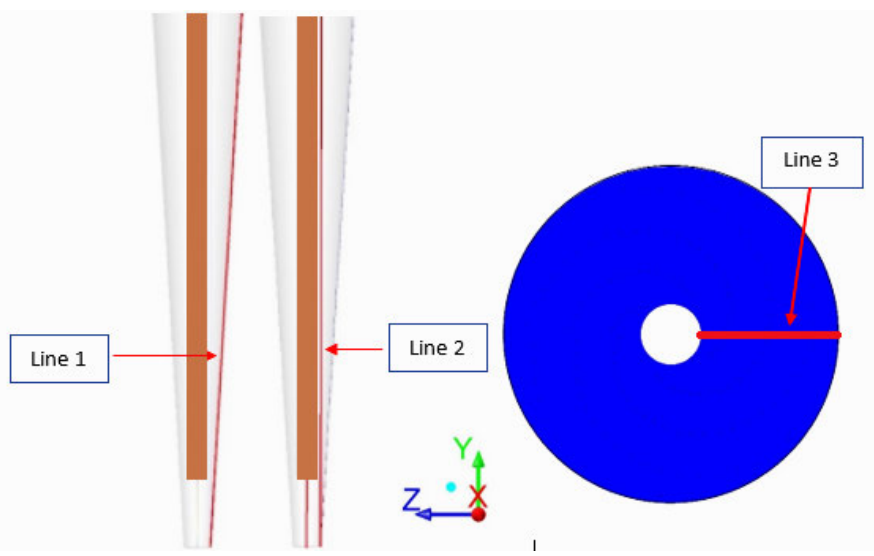

Figure 4. Data retrieval position using line/rake for quantitative data Shear stress (left), dynamic pressure and velocity (right), outlet velocity (Line 3 )

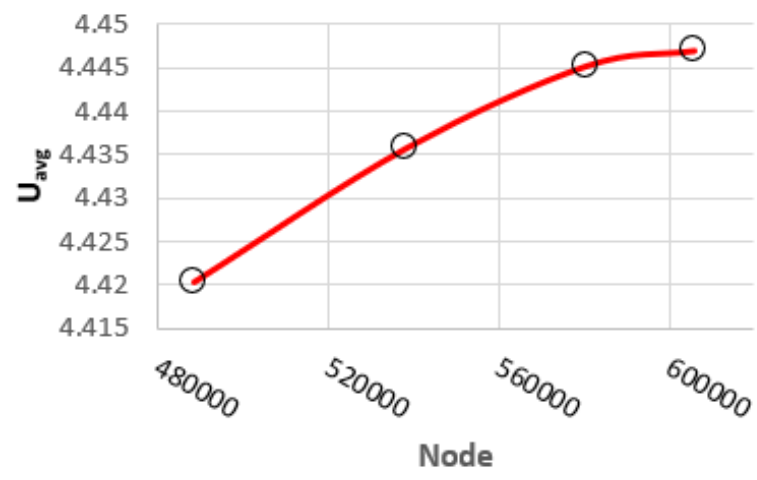

Figure 5. Grid independence reviewed at y $=1 \mathrm{~mm}$ 


\section{Results and Discussion}

3.1. Root canal length variations by using Positive pressure method

The velocity distribution at positive pressure with root canal length of $17.5 \mathrm{~mm}, 20.5 \mathrm{~mm}$, and $21 \mathrm{~mm}$ is the same. The open-ended needles created a jet to the apex and maximum irrigant replacement [7]. It can be seen that the distribution of velocity taken in each length of the root canal has an increase in velocity caused by the acceleration of gravity. After the fluid exits the needle, a very significant decrease in velocity is seen. This is caused by sudden enlargement, which suddenly enlarges causing a decreasing velocity. Next, the flow to the apical or base of the root canal with a high velocity so that there is backflow. This backflow occurs because the fluid collides with the wall so that the flow is in reverse direction then reduces velocity of the fluid going to the apical area. Illustration of backflow that occurs in the root canal can be seen in Figure 6. It can be seen that each root canal used has the same velocity profile. Vector velocity appears in areas close to the wall. They have a low velocity, while far from the wall have high velocity.

In Figure 7, a flow graph turns out the root canal. The direction of fluid reverses due to the impact on the wall of the root canal, which then flows to the reverse direction. It can be seen that the peak increase in fluid velocity is reversed with a value of $\pm 1.71 \mathrm{~m} / \mathrm{s}$ for each sample, and then the flow drops again until it exits the root canal. The decrease is very significant, and even the decrease is only $14 \mathrm{~mm}$ distance to $0.1 \mathrm{~m} / \mathrm{s}$ velocity. Analysis of the axial y component of irrigant velocity in the apical part of the canal as a function of the distance from the WL provided a more detailed overview of irrigant replacement, which is considered clinically significant for velocities greater than $0.1 \mathrm{~m} / \mathrm{s}[8]$. The replacement of irrigant extended further. This decrease occurred because of changes in the cross-sectional area flowed by the fluid. In addition, the fluid is against the direction of gravity so that its velocity decreases.

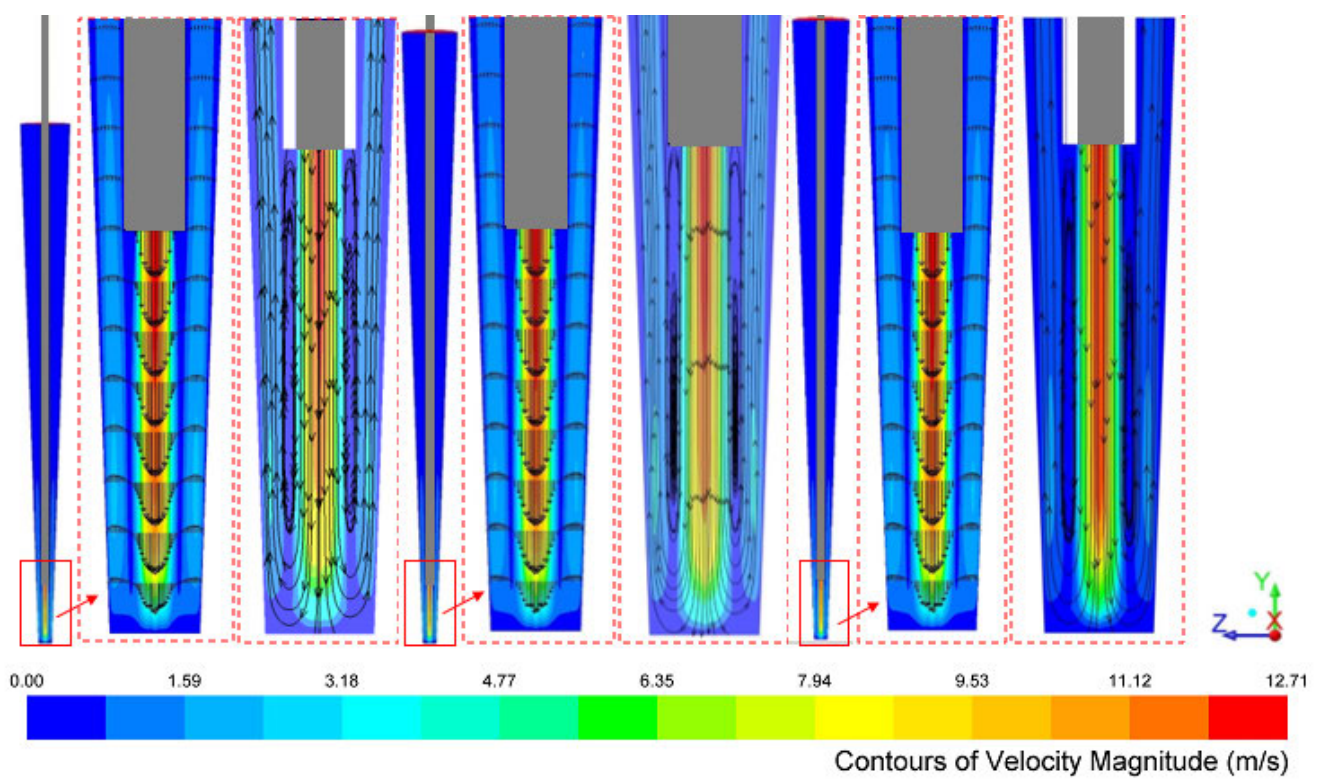

Figure 6. Contour velocity with vectors and pathlines

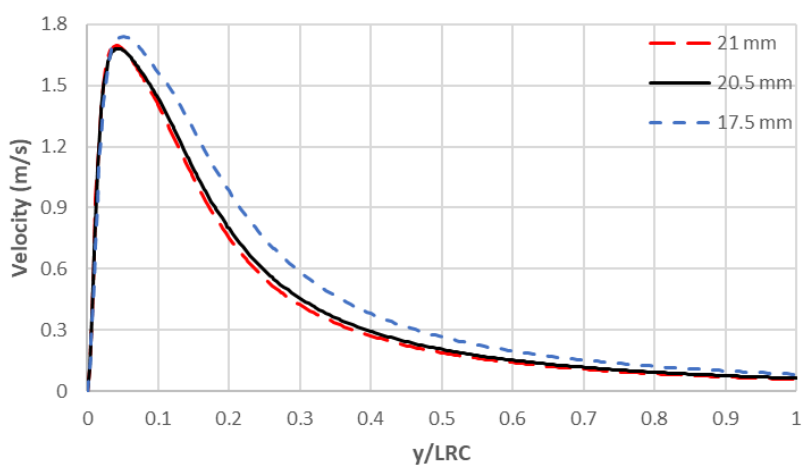

Figure 7. Graphic velocity distribution in each root canal 
3.2. Root canal length variations by using negative pressure method

In general, the open-ended needles created a jet toward the apex and maximum irrigant replacement [7]. The velocity distribution at negative pressure is more likely to be distributed evenly to the root canals. This happens because the distance between the apical and the tip of the needle is so far away, so there is no impact with the wall at high velocity which results in the end of the root canal is not exposed too heavily. In addition, the backflow occurs in the negative pressure method longer than the positive pressure method. This is illustrated in Figure 8 .

Based on Figure 8 viewed from the fluid mechanics, the flow is formed due to the difference in cross-sectional area or sudden enlargement. This causes a circular flow (vortex) on the outside. The flow is seen in the pathline, the vortex along between the apical and the tip of the needle.

Based on Figure 9, the graph shows that vary the length of the root canal has an impact on the negative pressure method. At the root canal length of $20.5 \mathrm{~mm}$, the flow to the outlet reaches $2 \mathrm{~m} / \mathrm{s}$, while at the root canal length, $22.5 \mathrm{~mm}$ is lower.

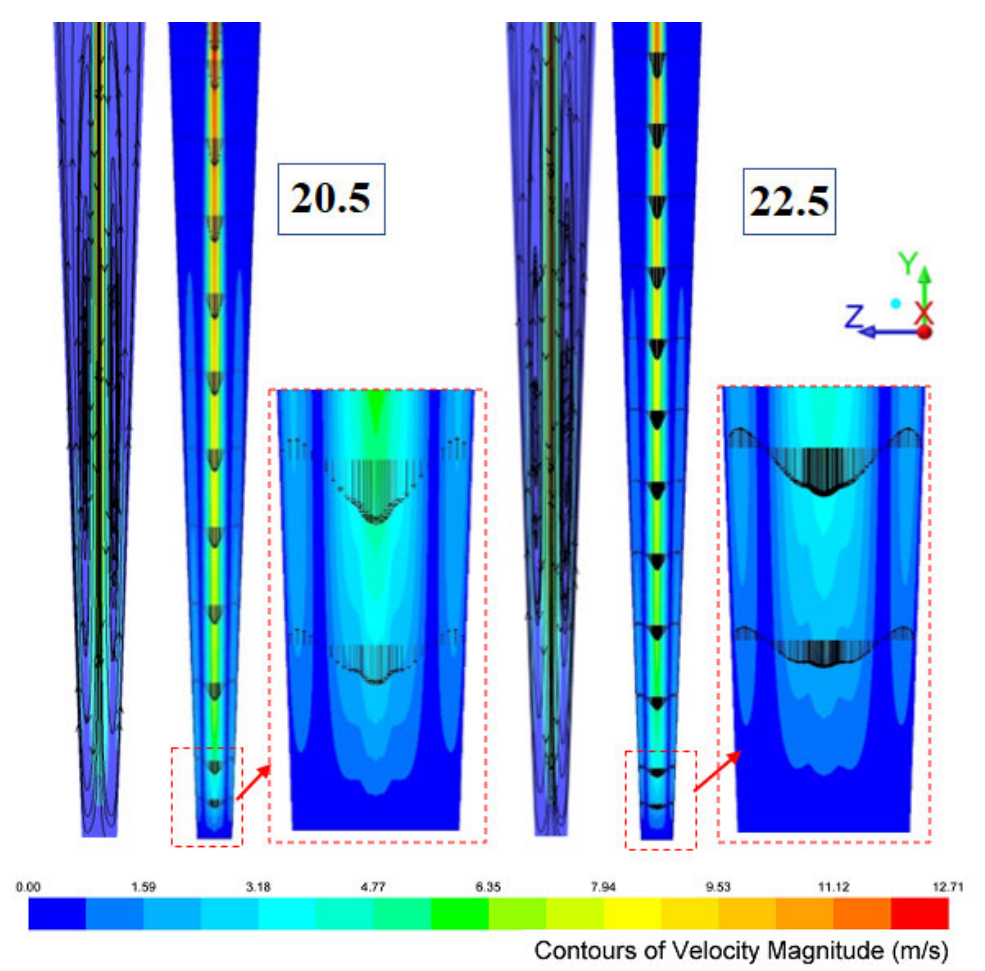

Figure 8. Contour velocity with vector and pathline negative pressure methods (a) root canal length $20.5 \mathrm{~mm}$ (b) root canal length $22.5 \mathrm{~mm}$

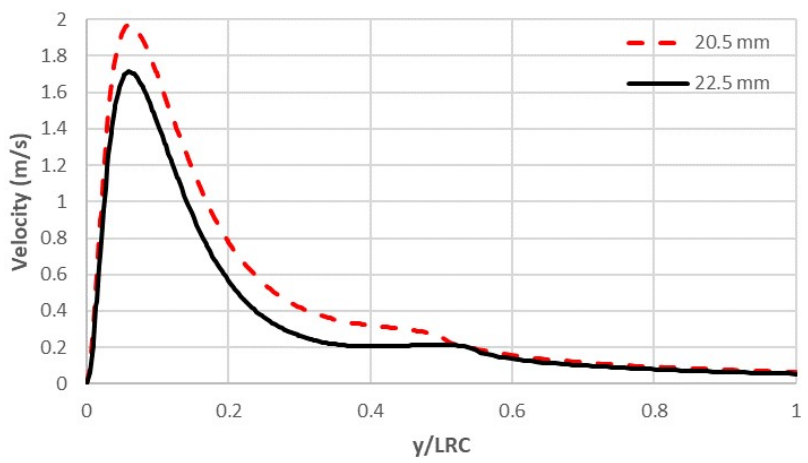

Figure 9. Velocity distribution of the negative pressure method 


\subsection{Comparison values on the apical}

This section will explain the values of velocity, dynamic pressure, and wall shear stress by comparing the same root canal length, that is, the root canal $20.5 \mathrm{~mm}$. In the positive pressure method the insertion length is up to 2 $\mathrm{mm}$ from the apical, whereas at negative pressure as far as $10 \mathrm{~mm}$ from the root canal outlet. Then the results of the simulation taken are at the base of the root canal or apical $\mathrm{y}=0 \mathrm{~mm}$ to compare the two methods. These differences are reflected in the numbers with their respective units so that they know the difference between the two methods. The results of the comparison are summarized in Table 2

From the results in Table 2 it can be seen that the dynamic pressure that hits the apical wall is very large so that it can cause accidents in irrigation of the root canal, whereas the negative pressure method is relatively safer than the positive pressure method because the dynamic pressure that occurs in the root canal is relatively small [9]. Reviewed from the wall shear stress that occurs at the base of the root canal, the positive pressure method is also greater than the negative pressure method. In addition, in terms of the maximum velocity that spread in the apical wall the positive pressure method is also greater than the negative pressure method. However, the negative pressure method still qualifies in the exchange of irrigation or debris removal, becauser the exchange of irrigation in the root canal occurs if the velocity is above $0.1 \mathrm{~m} / \mathrm{s}$ [8]
Table 2. Comparison values that occur in the apical region

\begin{tabular}{|c|c|c|c|c|}
\hline Methods & $\begin{array}{c}\text { Mean } \\
\text { Dynamic } \\
\text { Pressure (Pa) }\end{array}$ & $\begin{array}{c}\text { Mean } \\
\text { Wall Shear } \\
\text { Stress (Pa) }\end{array}$ & $\begin{array}{c}\text { Max } \\
\text { Velocity } \\
(\mathrm{m} / \mathrm{s})\end{array}$ & $\begin{array}{c}\text { Farthest } \\
\text { Distance } \\
(\mathrm{mm})\end{array}$ \\
\hline Positive & 581.97 & 36.66 & 2.21 & 0 \\
\hline Negative & 43.52 & 11.01 & 0.43 & 0 \\
\hline
\end{tabular}

\subsection{Comparison of Velocity Contours}

The difference between the two methods is the placement of the needle distance from the apical and the outlet. At the outlet, the positive pressure method is allowed to flow out by itself, and however, at the negative pressure method, the spray is sprayed into the vacuum root canal $-20 \mathrm{kPa}$ by the suction pump [10].

The difference in the method results in differences in many things, such as vortex, backflow length, and velocity distribution, as shown in Figure 10. In the positive pressure method, it is seen that the fluid hits apically at such a great velocity that the flow reverses. Then the length of the vortex formed in the positive pressure method is shorter and wider. The difference causes this in crosssectional area or sudden enlargement, which is smaller than the negative pressure method. In addition, the length of backflow found in the positive pressure method can only be up to $6 \mathrm{~mm}$ from apical.

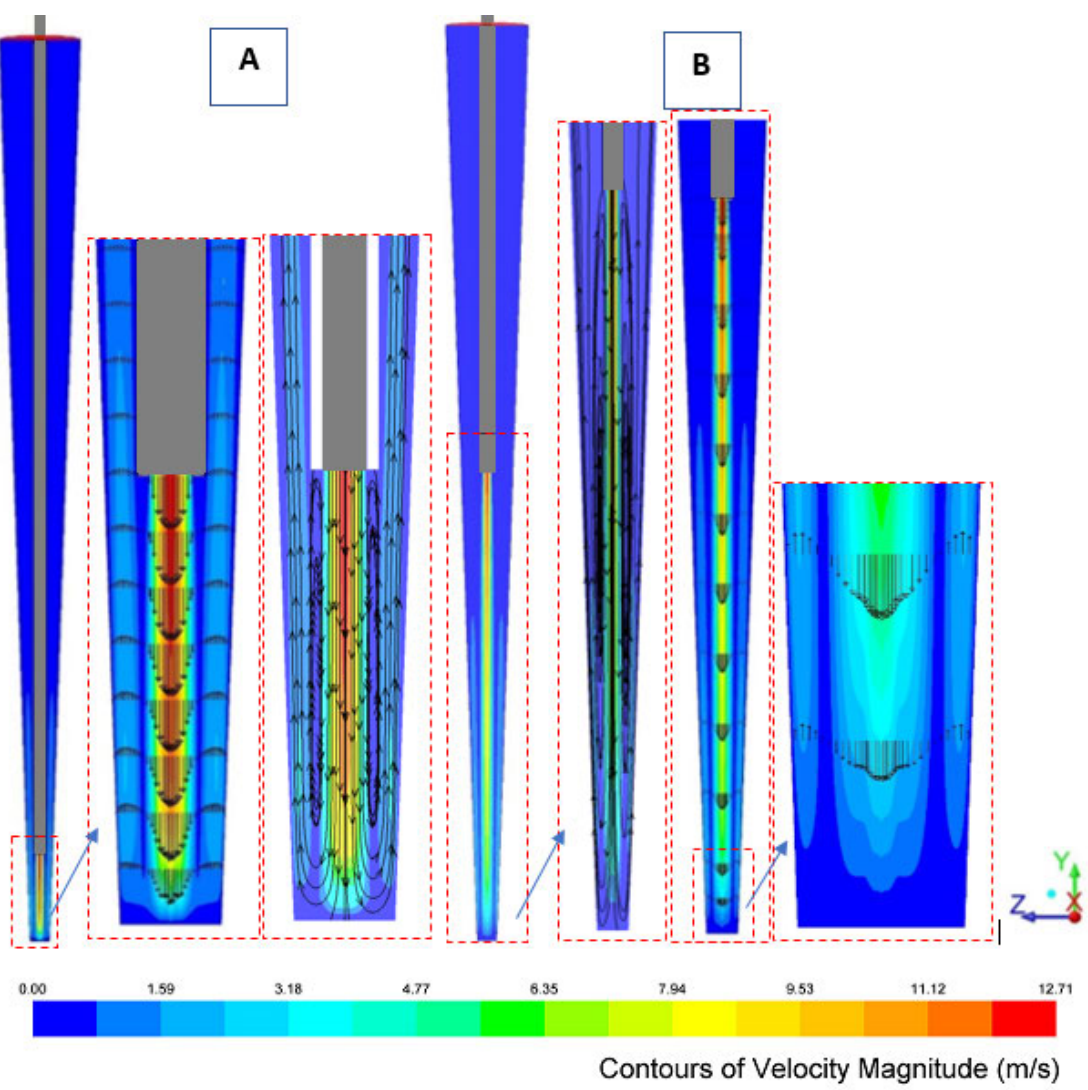

Figure 10. Contour velocity of the two methods (a) positive pressure method (b) negative pressure method 
This difference observes in terms of velocity that comes out at the outlet area as a condition of the minimum velocity for irrigant replacement or removal of debris. The minimum velocity for irrigant replacement is above $0.1 \mathrm{~m} / \mathrm{s}$ [7]. However, the two methods do not meet the requirements for irrigant replacement in the outlet area; this is illustrated in the graph in Figure 11 .

\subsection{Comparison of Dynamic Pressure Contours}

The difference in dynamic pressure is measured at the highest value of dynamic pressure generated in both methods. From the tabulated data, there is a small gap difference of dynamic pressure readings produced by these two methods in which $85,101.80 \mathrm{~Pa}$ is generated from the negative pressure method while a slightly higher value of 85,569.50 Pa obtained from the positive pressure method. The color portrays in the diagram for both methods is not highly visible due to slight differential pressures produced, as shown in Figure 12. Besides, the distribution of dynamic pressure in the positive method displays a red dynamic pressure contour approaching to the root canal/apical, which later changes in color gradually due to the collision with the apical wall. On the other hand, the negative pressure method has an evenly distributed dynamic pressure distribution along the root canal, in which the color will go down according to the scale after it passes through the needle.
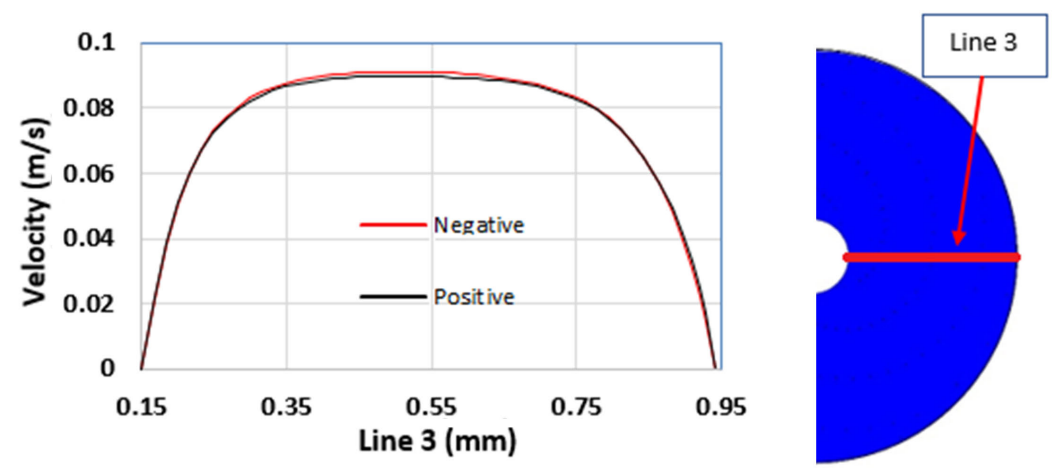

Figure 11. Comparison of velocity in both methods

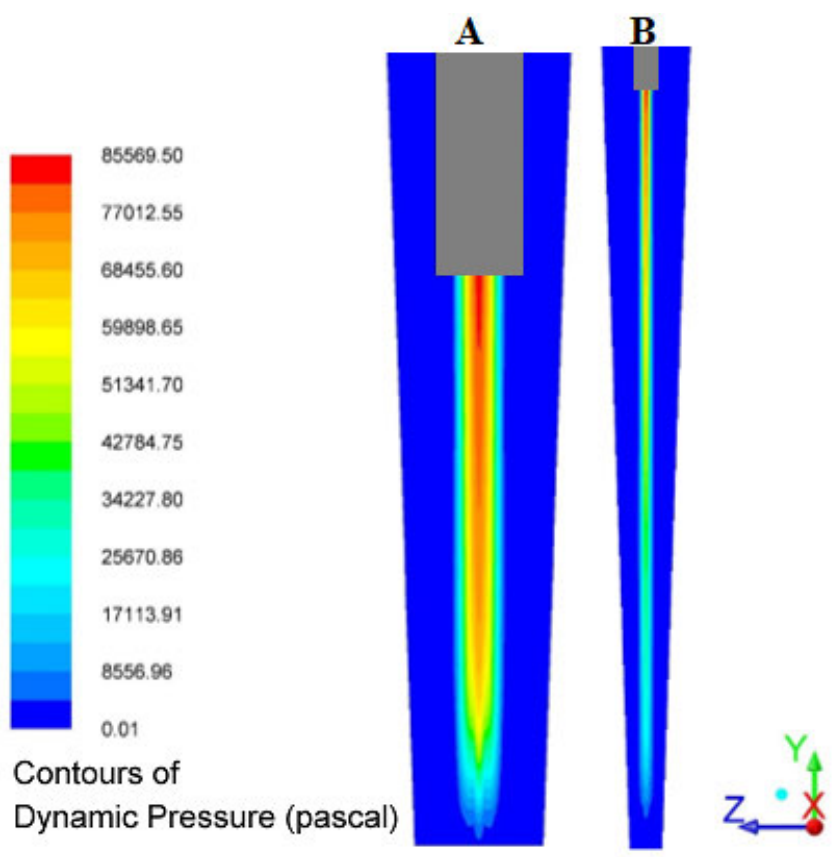

Figure 12. Visualization of dynamic pressure contours in both method (a) positive pressure method and (b) negative pressure method 
Based on Figure 13, the graph shows that the dynamic pressure returning to the outlet is higher in the negative pressure method. This is related to the velocity that occurs in the area. The peak of dynamic pressure leading to the outlet on the negative pressure method is $\pm 2,100 \mathrm{~Pa}$, while the positive pressure method is $\pm 1,500$ $\mathrm{Pa}$. After reaching the peak point, the dynamic pressure will drop simultaneously to the outlet.

\subsection{Comparison of Shear Stress Contours}

The positive pressure method has higher shear stress than the negative pressure method. This can be seen in Figure 14 It appears that the positive pressure method has a shear stress scale color that is red near the apical, and only green color appears in the negative pressure method. Moreover, it can also be seen that the shear stress in the positive pressure method is located near to the root canal or apical whereas, it only occurs along the root canal in the negative pressure method. Furthermore, on the apical wall of the root canal, the diagram also shows that the wall in the positive pressure method has shear stress in the area. However, the negative pressure method shows only dark blue color appears when it is viewed from api$\mathrm{cal}$, which indicates that the readings using the negative pressure method are not readable.

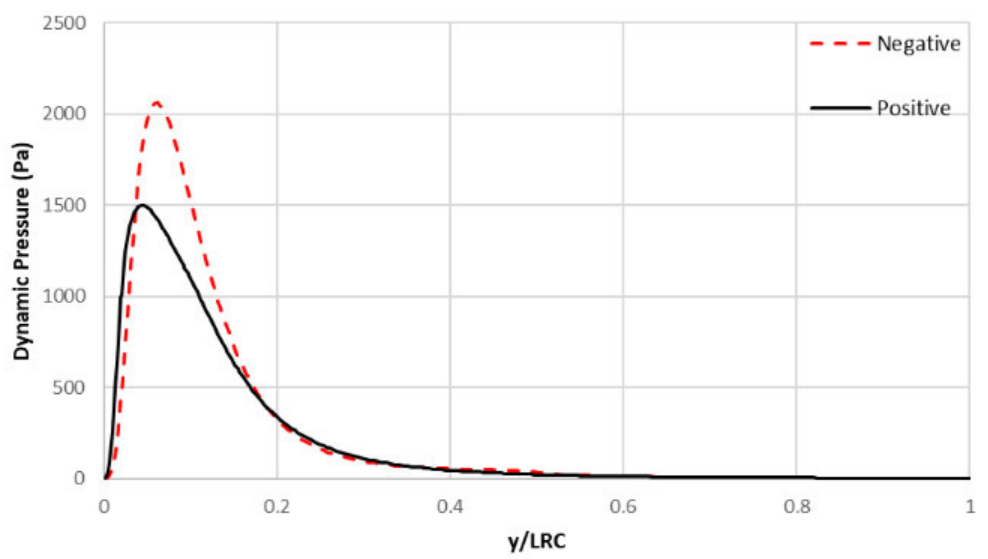

Figure 13. Comparison of dynamic pressure in the two method

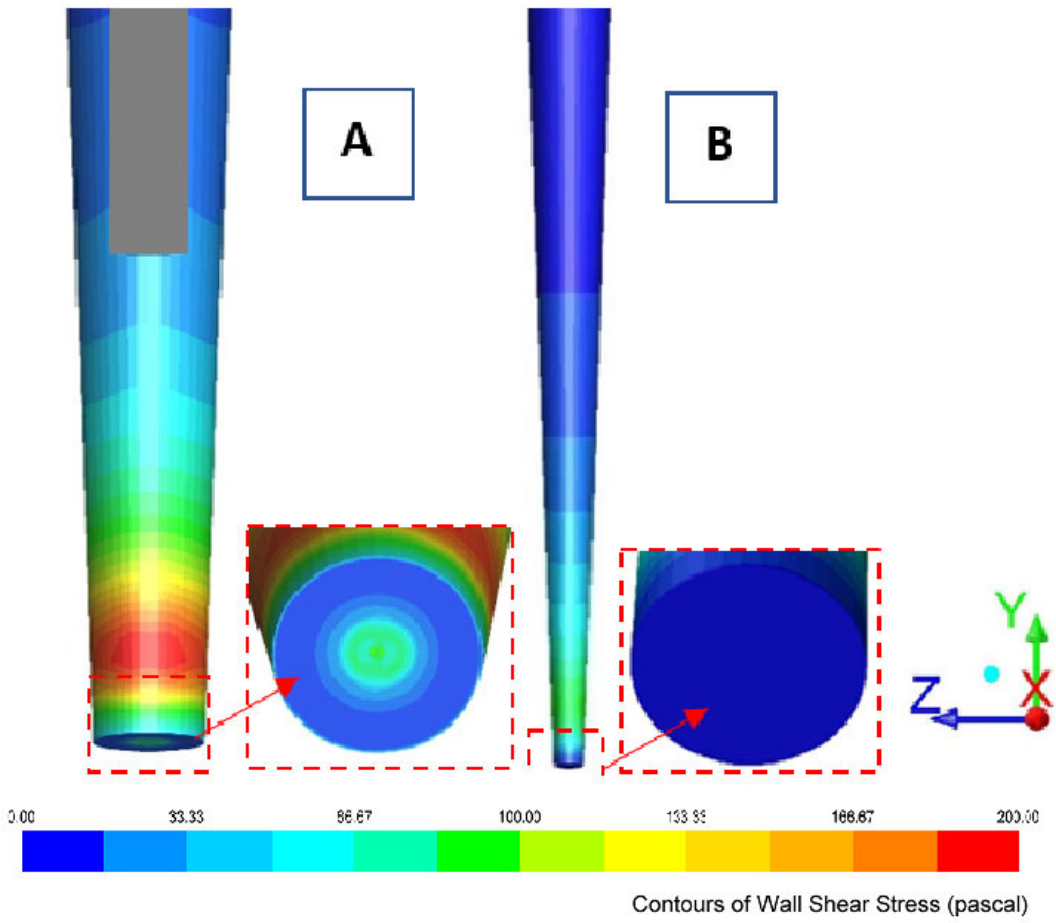

Figure 14. contour shear stresses of the two methods (a) positive pressure method (b) negative pressure method 
Apart from visualization, the comparison of the shear stress distribution between the positive and negative pressure methods is also displayed in graphical. The graph in Figure 15 shows that the differences lie in the highest value of shear stress that occurs in the root canal. The positive pressure method has higher shear stress of $\pm 180 \mathrm{~Pa}$, while the negative pressure method is only $\pm 120 \mathrm{~Pa}$ in which the shear stress contour in Figure 14 on the positive pressure method has a red color because of the highest scale used $200 \mathrm{~Pa}$. In addition, shear stress values in the positive pressure method are mostly found in the apical wall or root canal bottom, while in the negative pressure method, the shear stress found in the apical wall is only from $0-20 \mathrm{~Pa}$, so there is no presence of contours which are visible on visualization with a scale of $200 \mathrm{~Pa}$. Next, there is a sharp increment value of wall shear stress obtained in the negative pressure method before it starts to gradually decrease as it passes through the tip of the needle. At some point, the graph of both methods shows a trend in which the values are steadily decreasing throughout the output or outlet.

The correlation the results of wall shear stress with cleaning root canal irrigation is with a high value of the wall shear stress causes a greater flow momentum, so it can lift debris contained in the root canal [11]. From Figure 15, it is found that the positive pressure method is more effective in the apical region but cannot reach the outlet of the root canal [2]. However, In the negative pressure metcanhod, wall shear stress is more evenly distributed up to the middle of the root canal, so it is better than the positive pressure method.

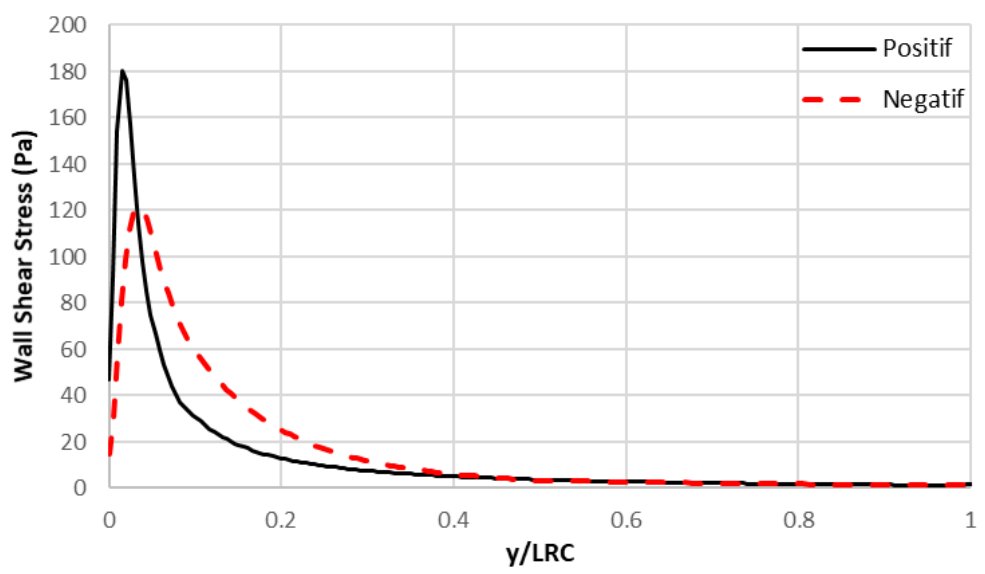

Figure 15. Graphic comparison of wall shear stress in both methods

\section{Conclusions}

The open-ended needles in positive pressure method and negative pressure method created a jet toward the apex and maximum irrigant replacement [7], because irrigant replacement occur when the velocity is above $0.1 \mathrm{~m} / \mathrm{s}$ [8]. However, the difference between the two methods is safety after reviewing the apical area [9]. It is found that the dynamic pressure in the positive pressure method was very high that made the possibility of accident occurring in root canal irrigation greater than the negative pressure method. In addition, the effectiveness of root canal irrigation for the negative pressure method is longer than the positive pressure method. Next, the shear stress that occurs in the positive pressure method is spread in the root canal base area, whereas in the negative pressure method, it spreads more evenly along the root canal. This results in greater flow momentum, which can then increase debris. So it was found that the negative pressure method can lift debris higher than the positive pressure method. In addition, suggestions for further research are to use granular flow simulations in order to display lifted particles, study the effect of variations in the position of an off-center needle, and use ultrasonic mothod.

\section{References}

[1] E. Park, Y. Shen, M. Khakpour, and M. Haapasalo, "Apical pressure and extent of irrigant flow beyond the needle tip during positive-pressure irrigation in an in vitro root canal model," Journal of endodontics, vol. 39, no. 4, pp. 511-515, 2013.

[2] Y. Fukumoto, I. Kikuchi, T. Yoshioka, C. Kobayashi, and H. Suda, "An ex vivo evaluation of a new root canal irrigation technique with intracanal aspiration," International Endodontic Journal, vol. 39, no. 2, pp. 93-99, 2006.

[3] C. Boutsioukis, E. Kastrinakis, T. Lambrianidis, B. Verhaagen, M. Versluis, and L. van der Sluis, "Formation and removal of apical vapor lock during syringe irrigation: a combined experimental and computational fluid dynamics approach," International endodontic journal, vol. 47, no. 2, pp. 191-201, 2014. 
[4] P. Li, D. Zhang, Y. Xie, and J. Lan, "Numerical investigation of root canal irrigation adopting innovative needles with dimple and protrusion," Acta of bioengineering and biomechanics, vol. 15, no. 1, 2013.

[5] K. Gulabivala, Y. Ng, M. Gilbertson, and I. Eames, "The fluid mechanics of root canal irrigation," Physiological Measurement, vol. 31, no. 12, pp. R49-R84, 2010.

[6] T. Kocharian, "Root canal irrigation-an engineering analysis using computational fluid dynamics," tech. rep., 2010.

[7] C. Boutsioukis, B. Verhaagen, M. Versluis, E. Kastrinakis, P. R. Wesselink, and L. W. van der Sluis, "Evaluation of irrigant flow in the root canal using different needle types by an unsteady computational fluid dynamics model," Journal of endodontics, vol. 36, no. 5, pp. 875-879, 2010.

[8] C. Boutsioukis, T. Lambrianidis, B. Verhaagen, M. Versluis, E. Kastrinakis, P. R. Wesselink, and
L. W. van der Sluis, "The effect of needle-insertion depth on the irrigant flow in the root canal: evaluation using an unsteady computational fluid dynamics model," Journal of Endodontics, vol. 36, no. 10, pp. 1664-1668, 2010.

[9] J. E. Chen, B. Nurbakhsh, G. Layton, M. Bussmann, and A. Kishen, "Irrigation dynamics associated with positive pressure, apical negative pressure and passive ultrasonic irrigations: a computational fluid dynamics analysis," Australian Endodontic Journal, vol. 40, no. 2, pp. 54-60, 2014.

[10] M. R. Ektefaie, "Point of care," Journal of the Canadian Dental Association, vol. 71, no. 7, 2005.

[11] D. Šnjarić, Z. Čarija, A. Braut, A. Halaji, M. Kovačević, and D. Kuiš, "Irrigation of human prepared root canal-ex vivo based computational fluid dynamics analysis," Croatian medical journal, vol. 53, no. 5, pp. 470-479, 2012. 